\title{
Urban Development Change as a Response to Information Technology
}

\author{
Janiece Upshaw \\ UNT Dallas
Theodore Larson
UNT Dallas
John Hubbard
UNT Dallas

\begin{abstract}
Change in information systems is a continuation of the development of technology, systems, and the urban environment throughout history. This paper puts this into perspective through three areas. By considering housing, jobs, and communication as a representative selection of functions based on Cooley's Theory of Transportation, it is possible to demonstrate the increasing need for a structured and deliberate consideration of the changing needs and opportunities inherent in a developing technology and communications-driven environment. These needs can be mirrored through housing, job, and communications needs as a progression of technology and through decentralization via communications and networks.
\end{abstract}

Keywords: urban development, information systems, communications, cities, history

\section{INTRODUCTION}

Modern information systems are not a new societal function within the broad spectrum of society. Just as Cooley acknowledges transportation as a key towards population centers (Hubbard, 2019), a corollary of his Theory of Transportation (Cooley, 1893) would suggest that communications are also key towards population centers. In response, we can trace a historical development of technological advances in housing (representing population), jobs (representing wealth), and communication (representing the break in transportation). While a number of urban development concerns could be considered, these are the most straightforward to consider within a technological capacity.

Just as the Romans developed roads that altered their ability to transport goods and messages, so more recent history has demonstrated the evolving nature of communication as a response to the development of new technologies. One principal example of this is in the communications methods that we use. Computers, information processing centers of any type, or managers at an operational or higher level are useless without an ability to transfer the results of the operations elsewhere. As a result, they are both driven by a need to increase the functionality and speed of communications and are, in turn, affected by said development. The primary result of this cycle is that information systems change and decentralize zones of high demand as the systems improve. 


\section{STRUCTURE}

There are a number of ways to classify communications systems. The cleanest way that is approachable from outside of the field of information systems is to do so by their structure. A broad metric for implementing this classification would be by complexity. Complexity, in this application, refers to a abstract topological approach wherein, the concern is which actors within the system can communicate with other actors in the system and what path the message takes. In this regard, communications systems can be classified as a point-to-point system, a hierarchical system, or a network - in order of increasing complexity.

In a point to point system, communications are handled from a single node to another node with no branching paths or alternate routes. An example of this would the common child's toy of two tin cans connected by a string. Sound waves pass from one tin can to the other across the medium of the string and either side can receive or transmit messages only from a single other source: the other tin can.

In a hierarchical system, the communications become more abundant. There can be several end nodes, which all transfer information through any one of several layers of intermediary nodes. This is often called a tree, as it mimics the structure of a tree: there's a central trunk, intervening branches, twigs, and finally leaves such that nutrients to the leaves and sugars from the leaves must pass through the trunk before being distributed to their destination. In this example, the leaves representing end points that can send or receive information. There is only one path between any one end node and any other end node.

A network seeks to address a fundamental flaw in the hierarchical system. Whereas a hierarchy is irreparably broken by the damage or destruction of the central 'trunk', a network attempts to provide multiple paths between nodes. Thus, a network becomes 'self-healing' to the extent that there is no critically important node such that damage or destruction cannot be routed around. As a result. The network requires a large amount of redundancy in infrastructure and in resources, but provides a more robust solution for the guarantee of transmission between any one point and another.

Some of the other classification systems may be of import solely with regard to the technology, but not necessarily at the highest levels of abstract planning. A system can be simplex, half-duplex, or full-duplex. That is, the messages can only go one way, can only go one way at a time, or can go both ways simultaneously across any given path. The technology can be classified by medium: word of mouth is the historical method; followed by written messages; or technological solutions such as copper electrical signals, optical signals over fiber, or wireless radio signals. In neither of these classification systems is the technology important for understanding and applying information systems to urban development, as the technology itself will change in time with only minor, iterative impact. However, while the structure itself will change over time, the increasing complexity of a system will likely have more profound effects, as the increase in connectivity has a lower bound of exponential increase in the number of available communications paths.

Counter to the previous statement, there is a potential imprecision in the term 'iterative'. In one sense, it is contrasting the comparatively geometric gains in complexity and utility through technological improvements. But in another sense, it could be noting the 'iteration' by time or technology generation, which is, itself, an exponential factor. The popular understanding of Moore's Law, for example, suggests that computers will double in speed every 18 months. This compounds loosely with the less popular Gilder's Law, which suggests that communication bandwidth doubles every 6 months. Regardless, whether the technology changes or the complexity of the network changes, the upper bound of the impact is still that the improvement of a communications system provides an improvement in the utility of the people who access that system.

\section{HISTORICAL EXAMPLES}

The growth of Information Technology can be viewed as the development of newer and more efficient forms of communication. History is rife with a number of these methods that provide an analogue to modern information systems and have led to changes in the distribution of urban centers. This brief outline of these changes conflates a bit the concept of urban design, urban development, and the distribution of population 
centers across a broader constituency. However, this is an acceptable approach within this abstract outlay, and further detail can be researched within the narrower confines of a specific topic.

\section{Transportation}

The earliest form of institutional communication would have been one village to another. Each village or city or city-state had a path that connected to each other one, with more or less maintenance depending on use. This point to point system was effective for handling individual communications, but were not conducive to larger organizations such as empires. The phrase 'all roads lead to Rome' was, in retrospect, a description of the Roman Empire's innovation of a system of roads that provided the ability for information to quickly be dispatched to all or some parts of the empire (there were similar innovations in Incan and Chinese history as well). Each road did not directly lead to Rome, as there were intermediary stops along the way. But the hierarchical design allowed for a quick distribution to any part of the network in a much more effective manner. This was then improved upon by the modern US Interstate highway system which provides multiple paths to get from any one location to another. This is vitally important in that it allows a dispersion between production and processing of raw materials, without having a single chokepoint. This highly networked system provides a modern convenience but requires a substantive overhead in maintenance.

A faster time scale analogue that illustrates the development of a technology over time would be the Railroad system. The famous Golden Spike that united the two ends of the Transcontinental Railroad was the first point to point transit from the east coast to the west coast of the United States. However, as traffic increased, it became apparent that multiple routes were needed. This was evident in the central hub cities that radiated out to other, local destinations. This hierarchical system was then added to during the great mobilization of World War II when military logistics realized that they would need multiple paths for multiple goods simultaneously accessing many different producers and shipping points.

The modern railroad system has not necessarily become a fully networked system of transport, as it was relatively quickly superseded by air shipment for many goods. This also illustrates that one category of technological improvement can be qualitatively supplanted by other categories. The Aerotropolis Model of economic development suggests a much more networked version of transportation.

\section{Communication}

While the transport of goods is conceptually similar to the transmission of information, the same pattern applies to communications patterns. Early methods of communications were focused on messengers who ran documents from one place to another and signal fire relays that would transmit simple messages from one point to another. But communications became much more interesting with the advent of the telegraph, which was another hybrid between point to point networks and hierarchical networks. The technology of the telegraph meant that every point along a given transmission line had access to every other point along the signal line. Because there was already an extant railroad system in place, the telegraph offices were placed along those lines. Both of these placements seem to justify Cooley's Theory of Transportation, which says that "Wealth and Population accrue at breaks in transportation.". In this case, the break in transportation would have been related to the distance that a steam train could travel before needing to stop and reload more fuel or water. In the case of telegraph lines, it was related to signal loss of the electrical signals over the copper lines. Coincidentally, those distances were approximately of the same magnitude, so there was a synergistic effect in collocating those facilities.

The broad implementation of the telephone system moved to a more hierarchical position. Early party lines notwithstanding, city hubs were responsible for routing signals to other cities. They were also responsible for routing signals within their areas of coverage to specific exchanges, which in turn were responsible for routing the telephone signal to individuals. This routing was done manually through operators, requiring a significant amount of time to connect long-distance phone calls. As the process was made more automated, the system still largely relied on the same hierarchical system of routing from one major node to another and then to lower level nodes on the hierarchy. 


\section{Modern Communication}

However, during the Cold War, DARPA (Defense Advanced Research Projects Agency) realized that there was a substantial problem with this model. Routing a call, possibly for military or defense purposes, from the east coast to the west coast typically required routing through specific nodes. Chicago, for example, was a primary hub through which many long-distance communications needed to be transferred. In the event of a nuclear strike on Chicago, there would be a total disconnection between east coast and west coast defense assets. They proposed that having a single route from one location to another was no longer sufficient. Instead, they proposed that a network of redundant connections be made between multiple different primary hubs so that in the event of a devastating strike in one location, communications could be routed alternatively. This was important for two reasons: it led to the development of DARPAnet as well as reducing the importance of communications sectors in Chicago and increasing the importance of communications sectors in previously unimportant locations. As a result, from a network perspective, Tulsa became just as important as Chicago.

This new DARPAnet eventually developed into the modern Internet that is the current standard for communications. The Internet is considered to be a 'self-healing' network. If, for example, there is a power outage in Chicago, communications will automatically be routed around Chicago. More importantly, it introduces a more analog measure of routing. If communications passing through Chicago are the fastest method to get data from one side of the country to the other, all data would try to flow through Chicago. As that happens and the physical lines become overloaded, that connection will slow down, meaning that passing through, again as an example, Tulsa will be faster and new communications will try to pass through there until there is a level of equilibrium between the speed of all available routes. This self-balancing effect becomes important in looking at how communications and information technology affect the development of cities and urban development.

\section{URBAN DEVELOPMENT}

If Urban Development can be reduced $a b$ absurdum to focusing on individual access to jobs, housing and communication, it becomes apparent that an increase in communication changes the levels of access to these basic needs. Just as national level networked routing will automatically self-correct until a form of equilibrium is reached, urban development also self-corrects with respect to transportation and communication.

Urban Development cannot be constrained to just jobs, housing, and communication. There are additional areas of interest, such as environmental management, identity, and aesthetic considerations. However, while framing the concern within the structure of Cooley, it makes the most sense to focus on the immediate areas. Increasing access to technology provides an increase in the distances these can be accessed from. There are exceptions, such as proximity to Wall Street (Beunza, 2004).

It is important in urban development to ensure that there is a robust system of networking in place to insure that no critical location is subject to risk. So, access to reliable electricity, phone, and internet means that urban planning has to avoid concentrations of high access and low access, resulting in a disparity in location value based on proximity to uncontrollable variables. As an aside, it is also important to note that there is a critical difference in systems that relate to physical utilities such as water and information utilities. Keisuke's (2008) Urban Environmental Management begins with a consideration of effective water management for sustainable buildings and broader natural water resources. This can be contrasted with Reynold, et al. (2019)'s treatment of Sand Branch, TX as an example of poor urban development relating to water as a resource.

\section{Housing}

There are a few aspects of this that apply to urban planning as a whole. The first is that real estate becomes decreasingly important as it relates to communications access as the quality of the network increases. In a point to point system, areas that are completely outside of the point to point relays are rendered inaccessible to communication or otherwise relegated to a secondary position as information takes 
longer to reach those locations. In a hierarchical system, this relegation is mitigated, but access to the communication nodes is still of primary importance. For example, in a historical phone or telegraph system, access or lack of access to nodes in the hierarchy present the same relegation. Further, because there is a necessary lag in communication time (implied, but unstated until this point), being further away from the highest tier of the network means that any of the communications will take longer. A modern example is with stock exchanges: as investment firms realize that access to the actual trading node (the central hub of the hierarchy), real estate becomes more valuable closer to the source. The difference may only be in the fractions of a second, but if an investment firm is intending to make thousands or millions of trades an hour, those fractions of a second become substantial.

It is only with a fully networked information system that these real estate prices become less of a fundamental concern. Because the traditional method of communication and goods transfer has typically been performed in a hierarchical manner (goods leave the processing plant and go to distribution points, and from there to their final destination; central communications backbones branch off into regional backbones and then to cities and individual locations), this access to more important locations has primarily driven growth within cities and other areas. The current trend, however, is towards a more distributed system: online shopping locations store goods in multiple places to reduce delivery times, high speed internet access becomes available to more and more suburban and rural areas, etc. This necessarily draws away from the importance of location in proximity to the central network locations as a factor in urban design leading to a more equitable distribution of opportunity and access.

\section{Jobs}

Another aspect is that as the connectivity between nodes approaches a more fully networked model, information and access become decentralized. This reduces the reliance on gatekeepers and other actors who might otherwise provide blocks to access.

Finally, this progression to a more fully networked model provides a substantial boost to the ability to communicate. A primary focus on this article has been on the historicity of the various models. Some of this is lost in the modern environment, to the benefit of all. A telephone client is not beholden to use the service of a single operator who happens to have the only hierarchical system that accesses their resident, but the more fully networked nature of the current model allows providers and consumers more choice of pattern, allowing more access to communication. Internet access allows employees to work remotely in part or in full, rather than be closely tied to their place of employment. Further, as the communication system goes globally, services such as Twitter, Discord, TikTok or others are allowing more broad communication between countries and cultures. While there is evidence that individuals self-select to maintain a comfort bubble of ideological safety, the opportunity to interact with their counterparts in other countries or cultures provides a means of interaction that has been historically absent.

\section{Communication}

This last, the global nature of intercommunicability, is - to a certain extent — a side effect of the fully networked situation. The intent is to provide a robust and decentralized communication medium. But that necessitates access to others with no central point of control, which will become a foundational need for communications and information systems as society progresses.

\section{CONCLUSION}

Information Systems, from a broad perspective, is the process of allowing communication from one person to another. It is a continuum between individuals talking with each other in person through automatic systems that digest and distribute large quantities of data several orders of magnitude faster, from one side of the planet to the other. From a public policy perspective, it is also a continuum between the communicability of individuals to the communicability of organizations.

The basic tenet is that communications systems will become more robust over time through a combination of more densely networking communication nodes and the supplantation of qualitatively 
superior communications methods as technology progresses. From a simpler, model-centric perspective, this can be easily viewed as a point to point communications system developing into a hierarchical system and then finally a fully networked medium.

There are two broad caveats to this: the first is that the process of progression to a more fully networked system is one that takes time to develop and will have forward and backward progress (from a localized time perspective), even though it will always be forward progress from a larger scope. The second is that while this is a concise and model-based description of the pattern and factors, there is always the opportunity for a new model to develop that improves on the fully networked model.

\section{REFERENCES}

Beunza, D., \& Stark, D. (2004, April). Tools of the trade: The socio-technology of arbitrage in a Wall Street trading room. Industrial and Corporate Change, 13(2).

Cooley, C. (1893). The Theory of Transportation. Baltimore, MD: Publications of the American Economic Association (9).

Hubbard, J., \& Larson, T. (2019, December). Cooley's Break in Transportation and the Physical-Digital Divide. Journal of Management Science and Business Intelligence, 4(2).

Keisuke, H. (2008). Urban Environmental Management and Technology. Springer Japan.

Reynolds, P., Upshaw, J., \& Larson, T. (2019, August). A Qualitative Study of an Environmental Justice Fight in a Freedman Community: A Content Analysis of Sand Branch, Texas. Journal of Theoretical and Philosophical Criminology. 